\title{
Bayesian spatiotemporal model of fMRI data using Transfer Functions
}

\author{
Alicia Quirós $^{a}$, Raquel Montes Diez ${ }^{a 1}$, Simon Wilson $^{b 2}$ \\ ${ }^{a}$ Departamento de Estadística e Investigación Operativa, Universidad Rey \\ Juan Carlos, Madrid, Spain \\ ${ }^{b}$ School of Computer Science and Statistics, Trinity College Dublin, Ireland
}

Corresponding author:

Alicia Quirós Carretero

Edificio Dept. II, Despacho 043

Universidad Rey Juan Carlos

C/ Tulipan, s/n

28933 Mostoles (Madrid)

Spain

e-mail: alicia.quiros@urjc.es

Phone: +3491 4888236

Fax: +34914887626

\footnotetext{
${ }^{1}$ raquel.montes@urjc.es

${ }^{2}$ simon.wilson@tcd.ie
} 


\section{Abstract}

This research describes a new Bayesian spatiotemporal model to analyse BOLD fMRI studies. In the temporal dimension, we describe the shape of the hemodynamic response function (HRF) with a transfer function model. In the spatial dimension, we use a Gaussian Markov random field prior on the parameter indicating activations that embody our prior knowledge that evoked responses are spatially contiguous. The proposal constitutes an extension of the spatiotemporal model presented in a previous approach [Quirós, A., Montes Diez, R. and Gamerman, D. (2010). Bayesian spatiotemporal model of fMRI data, Neuroimage, 49: 442-456.], offering more flexibility in the estimation of the HRF and computational advantages in the resulting MCMC algorithm. Simulations from the model are performed in order to ascertain the performance of the sampling scheme and the ability of the posterior to estimate model parameters, as well as to check the model sensitivity to signal to noise ratio. Results are shown on synthetic data and on a real data set from a block-design fMRI experiment, showing good performance in the detection of activity and significant flexibility in the estimation of the HRF.

Key words: Bayesian analysis, fMRI, HRF estimation, MCMC, spatiotemporal model, transfer functions 


\section{Introduction}

In this paper we propose a new model for estimating the hemodynamic response function (HRF) and determining which parts of the brain show activation in response to a stimulus in BOLD fMRI data. The proposal constitutes an extension of the spatiotemporal model presented in Quirós et al. (2010), offering more flexibility in the estimation of the HRF and computational advantages in the resulting MCMC algorithm.

By observing the relation between a stimulus paradigm (in an experiment) and the hemodynamic response based on BOLD effect (Ogawa et al., 1990), fMRI provides a measure of brain activation. Inference about brain activity in fMRI data is commonly addressed through the General Linear Model (GLM) analysis, introduced by Friston et al. (1995), in which a linear dependency of the BOLD signal and the HRF is assumed. Generally, the stimulus pattern is fit simply as a box-shaped wave, slightly delayed, in order to account for the lapse of time between the stimulus onset and the arrival of the blood to the activated area. This box-shaped wave is convolved with a HRF template for which several kernels have been considered, including Poisson (Friston et al., 1994), Gaussian (Friston et al., 1995) and gamma (Lange and Zeger (1997); Boynton et al. (1996)). The convolution approach is attractive for its simplicity. However, it imposes restrictions to the model, e.g. it forces antisymmetry and monotonicity on each half cycle, as mentioned by Crellin, Hastie and Johnstone in the published discussions of Lange and Zeger (1997). Moreover, to account for spatial fluctuations at the voxel level, the canonical HRF can be supplemented with its first and second derivatives to model differences in time (Friston et al., 1998). 
The fMRI data analysis involves the spatiotemporal relationship between a stimulus or cognitive task and the cerebral response measured with fMRI. As mentioned by Makni et al. (2008), the localisation of brain activation strongly depends on the modelling of the brain response and thus its estimation. The key point is therefore to tackle the two problems in a common setting (Makni et al., 2008), i.e. to set up a spatiotemporal formulation. Some of the spatiotemporal models proposed in the literature are Bowman (2007), Penny et al. (2005), Katanoda et al. (2002) and Woolrich et al. (2004a). Although all these models are based on convolution, the four of them present a different modelisation of the spatiotemporal correlation structure between voxels. Bowman (2007) incorporates a functionally defined distance metric into a parametric structure for spatial correlations within a ROI (with the difficulty of choosing the ROI and the functional distance between voxels in it) and includes temporal correlations between scans. Penny et al. (2005) propose a fully Bayesian model with spatial priors defined over regression coefficients of a GLM, using Gaussian Markov random fields (GMRF), and the errors are modelled as an autoregressive process. Katanoda et al. (2002) propose a spatiotemporal regression model for each voxel that involves the time series of the neighbouring voxels together with its own. Woolrich et al. (2004a) present a fully Bayesian approach, incorporating spatiotemporal noise modelling. A general problem of spatiotemporal models is the large number of parameters and, as a consequence, the great computational burden.

The Bayesian paradigm provides an appropriate framework for making inference using complex models and to overcome the multiple comparisons 
problem. It also constitutes a natural but rigorous theory for combining prior and experimental information. Most Bayesian approaches to the modelling of fMRI data use GMRF as prior distributions, in order to account for the spatial structure present in the data, e.g. Gossl et al. (2001) use GMRF to spatially regularise regression coefficients and Woolrich et al. (2004b) to spatially regularise AR coefficients. Moreover, several choices of the precision matrix of the GMRF prior on regression coefficients of a GLM-AR model have been considered in the Bayesian literature, these include uninformative priors (Penny et al., 2003), global-shrinkage priors Friston and Penny (2003) and Laplacian priors (Penny et al., 2005) among others. An interesting comparison of these priors can also be found in Penny et al. (2005).

Quirós et al. (2010) propose a Bayesian spatiotemporal model to determine active areas into the brain by merging the two stages of the model first proposed by Kornak (2000) and extended by Quirós et al. (2006). In the temporal dimension, the HRF is parameterised with an scaled Poisson probability density function. In addition, the delay of the HRF is not fixed in advance but modelled as an unknown parameter. In the spatial dimension, the authors use GMRF priors on activation characteristics parameters (location and magnitude) that embody the prior knowledge that evoked responses are spatially contiguous and locally homogeneous. In this way, smoothing is included as a part of the model and it is not left to a pre-processing step. Despite being spatiotemporal, the proposed model has a small number of parameters and all of them are interpretable.

In this work, we extend the spatiotemporal model described in Quirós et al. (2010) by including a more flexible modelling of the HRF. This is achieved 
by using a transfer function (TF) model to estimate the HRF. Transfer functions, also known as Finite Impulse Response (FIR) filters in the fMRI statistical analysis literature, were first used to model the HRF by Nielsen et al. (1997). Since then, they have proven to be a powerful and flexible tool to infer on the HRF shape. Goutte et al. (2000) adopt a transfer function model to estimate the HRF in fMRI, introducing a Gaussian process prior on the coefficients of the TF. The authors prove not just the ability of the FIR filter to recover the shape of traditional linear filters (Poisson, Gamma filter and Gaussian filters) but also that the smooth FIR filter is able to model additional features in real data, when the three traditional filter shapes fail. Ciuciu et al. (2003) generalise Goutte et al. (2000) in order to account for estimation of the HRF for any fMRI experiment. Makni et al. (2008) propose a parcel-based spatiotemporal approach under a Bayesian formalism. Regions are first identified as functionally homogeneous parcels in the mask of the grey matter and a region-based transfer function model of the HRF is adopted (Goutte et al. (2000); Ciuciu et al. (2003)). Detection is achieved by modelling activating, deactivating and nonactivating voxels through a mixture model (gamma-Gaussian) within each parcel.

The paper is arranged as follows: in the following section, we state the model and prior distributions of the parameters in the model and derive the corresponding posterior distribution. We examine the results obtained by applying the model to simulations, synthetic data and to real data in the results section. This is followed by the discussion and conclusions. 


\section{Materials and Methods}

\subsection{The Model}

Let us consider a fMRI experiment developed either under a block or a periodic event-related design. In a block design, two consecutive blocks (one with stimulus and one without) are called a cycle. In a periodic event-related paradigm, we define a cycle as the scans acquired between two stimuli. Let $C$ be the number of cycles in the experiment, $T$ the number of images in each cycle and $N \times M$ the dimension of each image. Notice that this is a $2 \mathrm{D}$ approach analysing axial slices. The whole data of the experiment may be given by

$$
y=\left\{y_{s, \tau, c}: s=1, \ldots, N \times M ; \tau=1, \ldots, T ; c=1, \ldots, C\right\}
$$

where $y_{s, \tau, c}$ is the value of voxel $s$, in the image number $\tau$ of the cycle $c$ and where each cycle $c$ is assumed to be identically distributed.

In this work, we revise the spatiotemporal model described in Quirós et al. (2010) by including a more flexible modelling of the HRF. The proposed model is as follows:

$$
y_{s, \tau, c}=b_{s}+z_{s} h_{\tau}\left(\nu_{s, k}\right)+\varepsilon_{s, \tau, c}, \quad \varepsilon \sim \mathcal{N}\left(0, \sigma^{2}\right)
$$

Field $b=\left\{b_{s}: s=1, \ldots, N \times M\right\}$ represents the baseline level for each voxel $s$.

Field $z=\left\{z_{s}: s=1, \ldots, N \times M\right\}$ is a binary random field, defining the presence (1) or absence (0) of activity. In practice, $z$-field is defined by thresholding a continuous field, w, i.e., $z_{s}=I_{\left\{w_{s}>0\right\}}$, and it provides a summary of location of activity. 
In order to allow more flexibility in the estimation of the HRF we propose the use of a transfer function model for the $\mathrm{HRF}, h_{\tau}\left(\nu_{s, k}\right)$, rather than a parametric curve as the Poisson density function employed in Quirós et al. (2010). Notice that by using a transfer function we do not impose a specific form on the HRF, leading to a greater ability to adopt any shape.

In a transfer function model an output series, $y_{t}$, is related with an input series, $x_{t}$, through a linear filter as

$$
y_{t}=\sum_{j=-\infty}^{\infty} \nu_{j} x_{t-j}+n_{t}
$$

where $\sum_{j=-\infty}^{\infty} \nu_{j} x_{t-j}$ is referred to as the transfer function or filter by Box and Jenkins (1976), and $n_{t}$ is the noise series of the system that is independent of the input series $x_{t}$. The coefficients in the TF model are often called the impulse response weights.

Here we use a causal and periodic (over cycles) transfer function model, so that the HRF is represented by

$$
h_{\tau}\left(\nu_{s, k}\right)=\sum_{k=1}^{K} \nu_{s, k} x_{\tau-k}, \quad \tau=1, \ldots, T,
$$

where the number of terms in the transfer function, $K$, is fixed to the number of images in a cycle $T$ and where $x_{\tau}$ is the stimulus paradigm, which according the design of the experiment, takes value one when the stimulus is on and zero when the stimulus is off.

The likelihood function is then derived and given by the following expres- 
sion

$$
\begin{aligned}
& f\left(y \mid b, z, \nu, \sigma^{2}\right)=\prod_{c} \prod_{\tau} \prod_{s} f\left(y_{s, \tau, c} \mid b_{s}, z_{s}, \nu_{s, k}, \sigma^{2}\right) \\
& \propto\left(\frac{1}{\sigma^{2}}\right)^{\frac{M N C T}{2}} \exp \left\{-\frac{1}{2 \sigma^{2}} \sum_{c=1}^{C} \sum_{\tau=1}^{T} \sum_{s=1}^{N M}\left[y_{s, \tau, c}-b_{s}-z_{s} h_{\tau}\left(\nu_{s, k}\right)\right]^{2}\right\} .
\end{aligned}
$$

\subsection{Prior distributions}

Under the Bayesian framework, it is necessary to specify prior distributions for the unknown parameters of interest.

Regarding the parameter representing the baseline level, $b_{s}$, we propose a Gaussian distribution, the usual conjugate prior distribution,

$$
b_{s} \sim \mathcal{N}\left(\mu_{b}, \sigma_{b}^{2}\right), \quad \forall s=1, \ldots, N \times M
$$

Note that, if a reference slice or an estimation of the baseline level for a particular fMRI study exists, it is easy to include this information in the model as the mean $\mu_{b}$, choosing the value of $\sigma_{b}^{2}$ according to our uncertainty about the estimation of the baseline.

Spatial connectedness (expected from activity) may be incorporated by, a priori, modelling the generator field of $z, w$ as a GMRF. Following Quirós et al. (2010) we use an improper GMRF model for w:

$$
\pi(w) \propto \exp \left\{-\frac{1}{2} \sum_{<s, t>}\left(w_{s}-w_{t}\right)^{2}\right\},
$$

where $\left\langle s, t>\right.$ denotes that voxels $s$ and $t$ are neighbours. Let $n_{s}$ denote the number of neighbours of voxel $s$ and $Q$ define the precision matrix associated 
to the distribution described in (7). Thus

$$
Q_{s t}= \begin{cases}n_{s} & s=t \\ -1 & <s, t> \\ 0 & \text { otherwise }\end{cases}
$$

from which follows directly that

$$
w_{s} \mid w_{-s} \sim \mathcal{N}\left(\frac{1}{n_{s}} \sum_{t \in \delta_{s}} w_{t}, \frac{1}{n_{s}}\right),
$$

where, $\delta_{s}$ denotes the neighbourhood of voxel $s$. In practice, we consider the first-order neighbourhood, also known as the 4-nearest neighbourhood. The conditional mean of $w_{s}$ is simply the mean of its neighbours, but does not involve an overall level. As stated in Rue and Held (2005), we can then concentrate on the deviation from any overall mean level without having to specify the overall mean level itself, i.e. the proportion of activated voxels. It is important to notice that after combination with the likelihood, marginal distribution for each $w_{s}$ becomes proper (see Quirós et al. (2010)).

It is desirable to impose smoothness in the coefficients of the TF, for stability of the solution. This is accomplished by a priori using a multivariate normal as prior distribution for $\nu_{s}=\left\{\nu_{s, 1}, \ldots, \nu_{s, K}\right\}$, i.e. by defining

$$
p\left(\nu_{s}\right) \propto \exp \left\{-\frac{1}{2}\left(\nu_{s}-\mu_{\nu}\right)^{\prime} Q_{\nu}\left(\nu_{s}-\mu_{\nu}\right)\right\},
$$

where the precision matrix $Q_{\nu}$ is defined in order to incorporate our prior beliefs of smoothness. Accordingly, $Q_{\nu}$ is the inverse of a covariance matrix, $Q_{\nu}=\Sigma^{-1}$, whose elements are given by a decreasing function of the distance between two coefficients of the TF. For instance, Goutte et al. (2000) propose

$$
\Sigma_{i j}=\kappa \exp \left\{-\frac{1}{2 l^{2}}(i-j)^{2}\right\},
$$


where the hyperparameters $\kappa$ and $l$ should be chosen to fulfill the desired smoothness level in the resulting hemodynamic response.

Finally, the variance $\sigma^{2}$, is also modelled by the corresponding conjugate prior distribution, i.e. an inverse gamma distribution

$$
\sigma^{2} \sim \mathcal{I} \mathcal{G}\left(g_{1}, g_{2}\right)
$$

where $g_{1}$ and $g_{2}$ should be chosen to describe prior knowledge, but if such information is not available $g_{1}, g_{2} \rightarrow 0$ would define a non-informative prior distribution for $\sigma^{2}$.

\subsection{Posterior distribution}

By using Bayes theorem, we combine the prior information with the likelihood and obtain the following expression for the posterior distribution of the parameters, given the data,

$$
p\left(b, z, \nu, \sigma^{2} \mid y\right) \propto f\left(y \mid b, z, \nu, \sigma^{2}\right) p(b) p(w) p(\nu) p\left(\sigma^{2}\right) .
$$

In equation (13), the likelihood function, $f\left(y \mid b, z, \nu, \sigma^{2}\right)$, is given by equation (5), and the prior distributions for $w$ and $\nu, p(w)$ and $p(\nu)$, correspond to equations (7) and (10), respectively. The equations of the prior distributions for the rest of the unknown parameters are given below:

$$
\begin{aligned}
p(b) & =\left(\frac{1}{\sqrt{2 \pi} \sigma_{b}}\right)^{N M} \exp \left\{-\frac{1}{2 \sigma_{b}^{2}} \sum_{s=1}^{N M}\left(b_{s}-\mu_{b}\right)^{2}\right\} \\
p\left(\sigma^{2}\right) & =\frac{1}{g_{2}^{g_{1}} \Gamma\left(g_{1}\right)}\left(\frac{1}{\sigma^{2}}\right)^{g_{1}+1} \exp \left\{-\frac{1}{g_{2} \sigma^{2}}\right\}
\end{aligned}
$$

In order to make inferences about the unknown parameters, we use MCMC sampling techniques. 
It is important to highlight here that by using the proposed conjugate priors, the resulting posterior distributions are of known form, allowing us to employ Gibbs sampler steps for all the parameters of interest. This results in a MCMC algorithm that is easier to implement and therefore an important computational improvement with respect to the MCMC algorithm used in Quirós et al. (2010), in which some Metropolis-Hastings steps were required.

\section{Results and Discussion}

\subsection{Simulation from the model}

In this section, we simulate data from model (2) in order to ascertain the performance of the sampling scheme and the ability of the posterior to estimate model parameters.

\subsubsection{Simulation procedure}

We simulate $C=10$ cycles of $T=20$ images of dimension $20 \times 20$. Field $z$ describes a centred disk of value 1, leading to 177 active pixels. We sample the $b$-field from a Gaussian distribution, $\mathcal{N}\left(500,10^{2}\right)$, for each pixel independently.

The coefficients of the TF are sampled following equation (10), for each pixel, where the mean is set to zero, $\mu_{\nu}=0$, and the precision matrix $Q_{\nu}$ is computed by inverting the covariance matrix, $\Sigma$, which is defined in equation (11). The values chosen for the hyperparameters are $\kappa=100$ and $l=3$. We

obtain a realisation from the required distribution following Rue and Held (2005). 
The stimulus paradigm defines a block-design in which the stimulus is presented during the ten first images of each cycle. As a consequence,

$$
x_{\tau}= \begin{cases}1, & \tau=1, \ldots, T / 2 \\ 0, & \text { otherwise. }\end{cases}
$$

Finally, in order to test the performance of the model with the increase of noise, we give $\sigma^{2}$ values 100, 1000 and 10,000, leading to three simulated data sets.

\subsubsection{Inference results}

We obtain 10,000 samples of the posterior distribution. We discard the first 5000 iterations as burn-in and make inference using the remaining 5000 samples. Convergence was assessed by calculating approximations to Geweke (1992) convergence statistics and by observing how the values of the parameters change over the course of simulation. Studying Geweke's statistic values for each parameter and each sample, there is no evidence that convergence has not occurred as their values lay inside the interval $(-2,2)$.

In order to ensure that the model recovers the simulated data, Figure 1 shows a scatter plot of the simulated data, $y$, versus the posterior mean estimate of $b+z x h(d)$, for the data set simulated with $\sigma^{2}=10,000$, showing a good image recovery for the three data sets.

Figure 2 represents the scatter plots of simulated values versus the corresponding posterior mean values for field $b$, for the three different values of $\sigma^{2}$. Notice that a correct estimation of the baseline level $b$ for all the values of $\sigma^{2}$ can be observed in Figure 2 .

More interestingly, by observing Figure 3, in which the posterior mean 


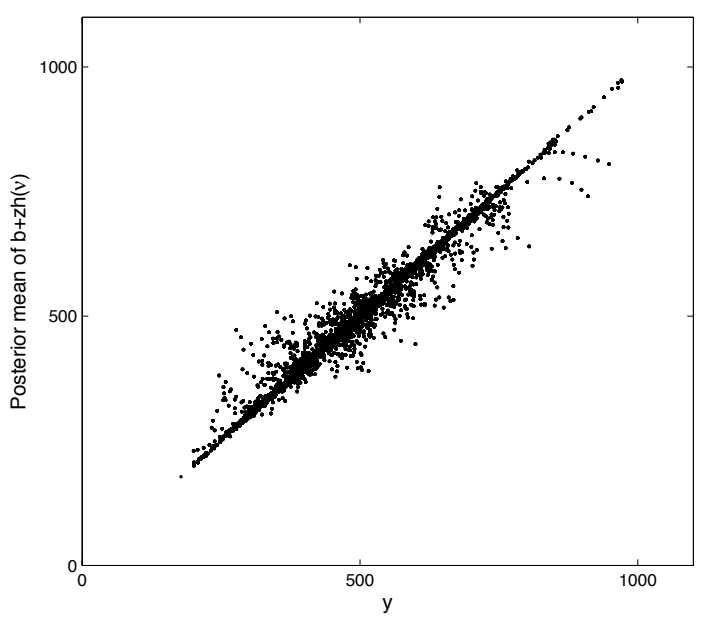

Figure 1: Simulated $y$ versus posterior mean estimate of $b+z h(d)$.

map of field $z$ is depicted, we notice that all active and non-active pixels are detected accurately by the posterior mean of $z$ for error variances $\sigma^{2}=100$ and $\sigma^{2}=1000$. However, we can also appreciate that as $\sigma^{2}$ increases, the number of misclassified pixels also increases.

Regarding the recovery of the TF, Figure 4 includes a scatter plot of the simulated TF, which is different for every pixel, versus the posterior mean map of $h_{\tau}(\nu)$, for each value of $\sigma^{2}$. Notice that the cloud of points becomes wider with the increase of noise. As expected, estimation of the TF is more accurate where less noise is present.

Provided that the level of noise is moderate, we can conclude that the procedure is capable of recovering the components of the model, the estimation is correct and the sampling procedure is working well. 


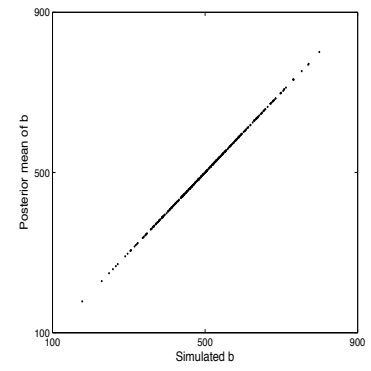

(a) $\sigma^{2}=100$

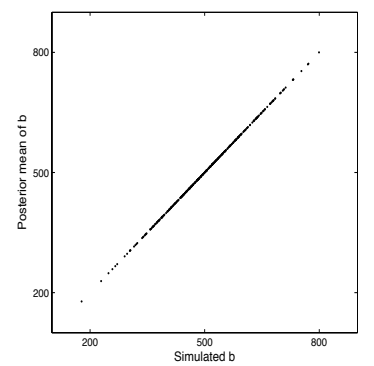

(b) $\sigma^{2}=1000$

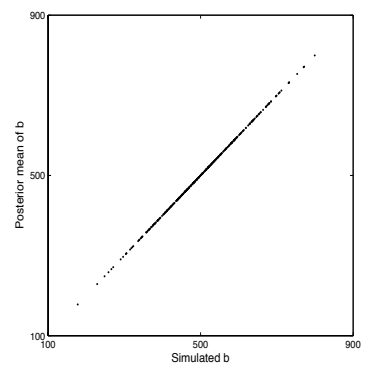

(c) $\sigma^{2}=10,000$

Figure 2: Scatter plot of simulated values versus posterior mean of $b$-field.

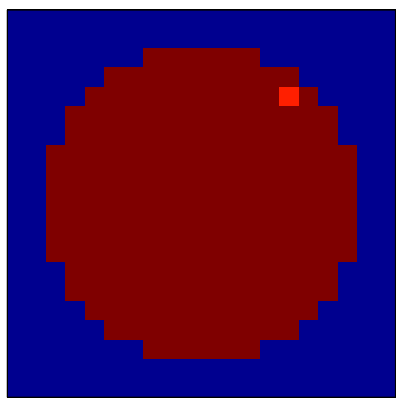

(a) $\sigma^{2}=100$

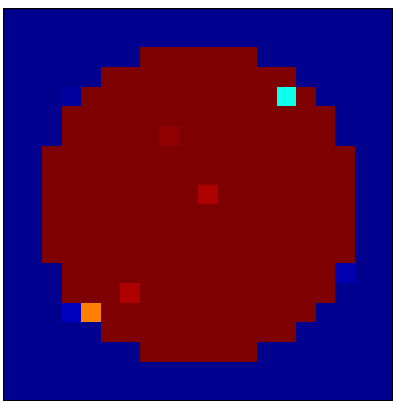

(b) $\sigma^{2}=1000$

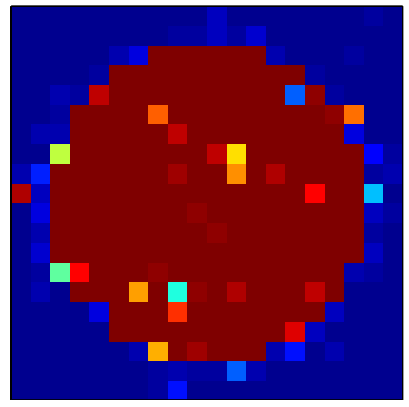

(c) $\sigma^{2}=10,000$

Figure 3: Posterior mean map of $z$-field.

\subsection{Synthetic data}

In this section, we simulate an fMRI study based on real data, in order to check the model applicability and sensitivity. We start the section explaining how the synthetic data are created, followed by the inference results.

\subsubsection{Synthetic data creation}

We create a synthetic data set from a real fMRI study, acquired under resting condition, with a $3 \mathrm{~T}$ scanner in the Ruber International Hospital of 


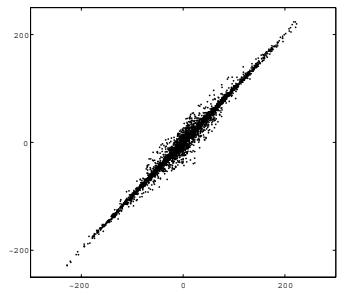

(a) $\sigma^{2}=100$

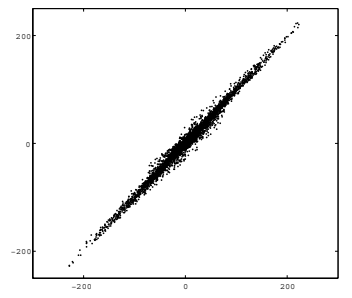

(b) $\sigma^{2}=1000$

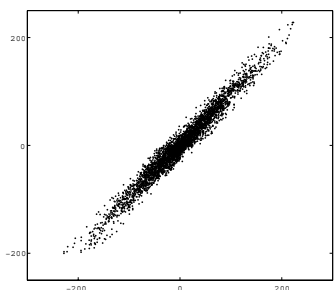

(c) $\sigma^{2}=10,000$

Figure 4: Scatter plot of simulated values of the TF versus posterior mean of $h_{\tau}(\nu)$-field.

\section{Madrid.}

The synthetic data are then simulated as follows

$$
y=\psi \odot(\alpha \otimes \rho)+\psi
$$

where $\otimes$ defines the Kronecker product.

$$
y=\left\{y_{s, \tau, c}, s=1 \ldots, N \times M ; \tau=1, \ldots, T ; c=1 \ldots, C\right\} \text { denotes the }
$$
resulting synthetic data.

$$
\psi=\left\{\psi_{s, \tau, c}, s=1 \ldots, N \times M ; \tau=1, \ldots, T ; c=1 \ldots, C\right\} \text { is a central slice }
$$
selected from the real fMRI rest study.

$\rho=\left\{\rho_{s}, s=1, \ldots, N \times M\right\}$ defines the spatial variation of activity. Here $\rho$ is a a binary on/off phantom showing different geometric figures to define activation regions. Figure 5 (a) shows the phantom used to simulate spatial variation of activity.

$\alpha=\left\{\alpha_{\tau, c}, \tau=1, \ldots, T ; c=1, \ldots, C\right\}$ defines the temporal variation of activity. Here, this activation pattern takes the shape of the convolution of a box car curve, $\varsigma_{\tau, c}$, with a gamma probability density function, $\gamma_{\tau}$, of shape parameter 2 and scale parameter 1 . In particular, we are interested in 
simulated activity intensity of $1 \%$ of the signal, as a consequence, we define $\alpha_{\tau, c}=0.01\left(\varsigma_{\tau, c} * \gamma_{\tau}\right) / \max _{\tau}\left(\varsigma_{\tau, c} * \gamma_{\tau}\right)$, where $*$ stands for convolution. Figure 5 (b) shows the temporal shape of the simulated HRF for a fixed $c$.

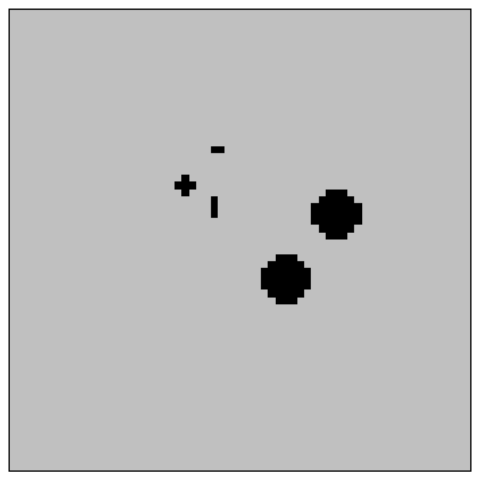

(a)

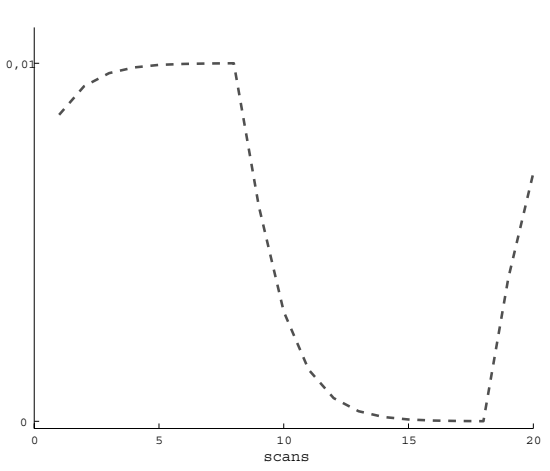

(b)

Figure 5: Activity phantom used to simulate the spatial variation of activity (a) and temporal shape of the response paradigm to a stimulus (b) in the synthetic data creation.

The result is a series of $300(C=15$ cycles of $T=20$ images each $)$ scans of $N \times M=64 \times 64$ voxels, that resembles a block-design fMRI experiment.

\subsubsection{Inference results}

We perform a total of 10,000 iterations, the first 5000 of which are discarded as burn-in. Subsequently, 5000 samples are used to make inference over the parameters. As in the previous section, vague priors are chosen for $b$, $\nu$ and $\sigma^{2}$ by setting large values for the corresponding variances and MCMC convergence was assessed by both visual inspection on the simulated chains and calculation of Geweke (1992) statistic, obtaining similar conclusions to those in the previous section. 


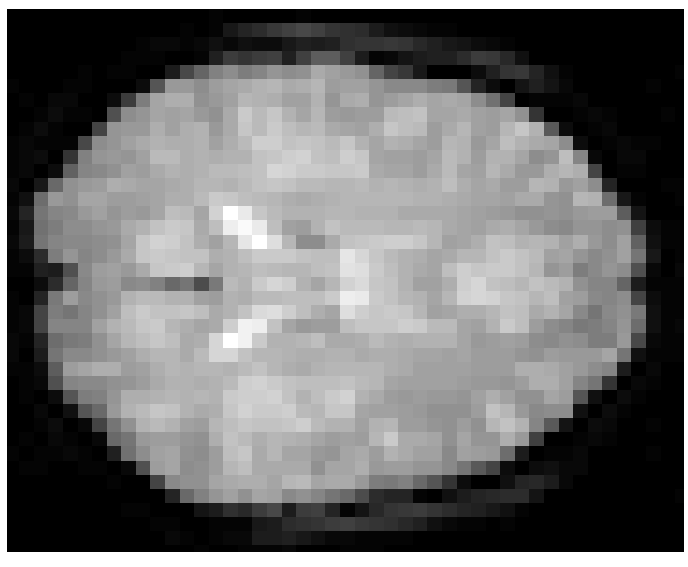

(a)

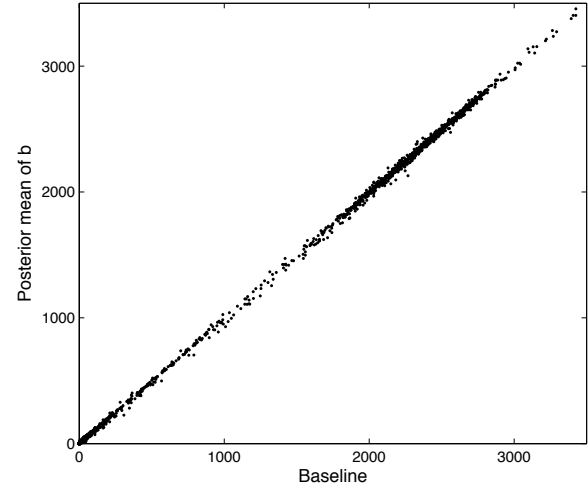

(b)

Figure 6: (a) Posterior mean of $b$ and (b) scatter plot of the baseline vs. the posterior mean of $b$.

Figure 6 shows results of the estimation of the baseline. Recall that it is expected that the posterior mean map of $b$ (Figure 6 a) resembles an image of the brain under resting conditions. In order to provide a graphical illustration of the accuracy of this estimation, Figure 6 (b) displays the scatter plot of the real baseline versus the posterior mean map of $b$, demonstrating an accurate performance of the estimation.

An important point to highlight is that by using a Bayesian framework, we are able to probabilistically quantify any event of interest. For instance, we may be interested in those areas which probability of activation is above a given percentage, $1 \%$ say. Following model (2), this is achieved by calculating

$$
P\left(\frac{z_{s} \max _{\tau} h_{\tau}\left(\nu_{s, k}\right)}{b_{s}}>0.01\right)
$$

where $\tau=1, \ldots, T$. It is interesting to notice here that an advantage of this calculation is the fact that it involves all parameters in the model, leading 
to a better estimate of the activity, for each voxel.

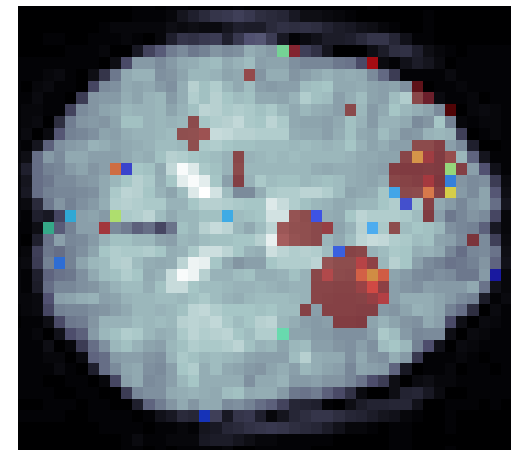

(a)

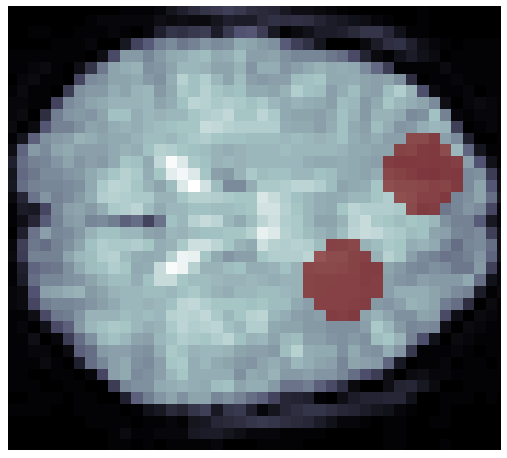

(b)

Figure 7: Posterior probability map that the increase of signal is greater than 1\% (a) and the same result for the model described in Quirós et al. (2010) (b).

Figure 7(a) shows the posterior probability map that the increase of signal is greater than $1 \%$. Note that most (90\% approximately) activated voxels are detected although activity is also shown in several (around 20) voxels outside active areas.

For comparison purposes, we also show the same PPM calculated by using the spatiotemporal model proposed in Quirós et al. (2010) (Figure 7b). Observe that in that case, the two large areas were perfectly defined and that activation in isolated voxels was totally ignored. We may therefore conclude that from a spatial point of view, the detection of activated areas favours the proposal of Quirós et al. (2010).

Nevertheless, recall that the objective of introducing transfer functions into the spatiotemporal model was to improve the estimation of the HRF when compared with the model proposed in Quirós et al. (2010) and, in general, with parametric approaches. For instance, Figure 8 shows the fit of 
one cycle for both the TF and the Poisson parameterisation of Quirós et al. (2010), in an active voxel. The convolved gamma curve used to create the synthetic data is also plotted. Although both approaches provide accurate estimations, it can be appreciated that the $\mathrm{TF}$ is able to better capture the shape of the gamma curve. Notice, for instance, that the restrictions imposed by the Poisson parameterisation do not allow the curve to model the final increase while the transfer function adopts a similar shape.

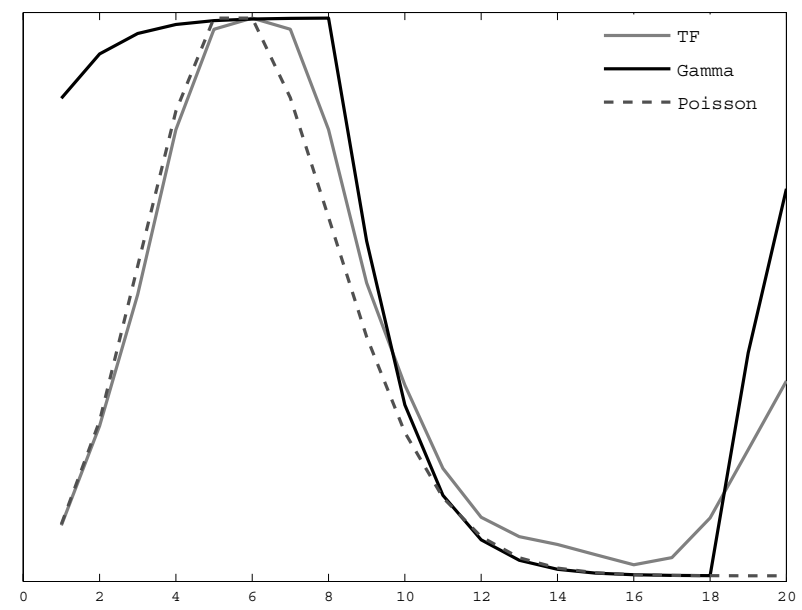

Figure 8: Estimation of the HRF for the TF and the Poisson parameterisation of Quirós et al. (2010), in an active voxel and one cycle of the convolved gamma curve used to create the synthetic data.

As already stated in Makni et al. (2008), the price to be paid for a flexible modelling lies in a loss of sensitivity of detection. That is, there is a trade-off between detection of activity and estimation of the hemodynamic response. 


\subsection{Real data}

For completion purposes, we apply the model to a data set provided by Ruber International Hospital. In this visual experiment, $6064 \times 64 \times 8$-scans are acquired, while the subject is submitted to a stimulus during images 11-20, 31-40 and 51-60. Therefore, we consider $C=3$ cycles of $T=20$ images each. The coil is moved as close as possible to the region of interest, in this case, the occipital cortex, which gives the very strong signal in the area. The scans are registered before statistical analysis and no smoothing is performed. For simplicity, graphical results are only provided for slices 3 and 5, although similar performance could be observed for the remaining slices.

Similar comments apply to those in the previous section regarding the elicitation of prior distributions and MCMC sampling settings. Analysis was carried out in a MacBookPro $(2.53 \mathrm{GHz}$ Intel Core Duo processor and a memory of 4 GB $1067 \mathrm{MHz}$ DDR3) with a Mac OS X Version 10.5.5. The MCMC scheme has been implemented in Matlab 7.7.0 requiring approximately $35 \mathrm{~s}$ for every 1000 iterations (around 6 minutes for the analysis of each slice).

In order to obtain a map of activity, using the posterior information provided by all parameters in the model, we calculate the posterior probability that the increase of signal is greater than 0.01, as described in the previous section. Figure 9 presents this PPM superimposed on a reference image from the study. As stated previously, this permits a quantitative characterisation of the activation pattern. For comparison purposes, we perform an analysis of these real data with SPM, using temporal basis functions (the canonical $\mathrm{HRF}$ and its temporal and dispersion derivatives) and a Bayesian estimation procedure with GMRF prior distributions for signal and noise. Figure 10 
shows the resulting estimate of activity By observing figures 9 and 10, we can appreciate that the two approaches show similar performances.

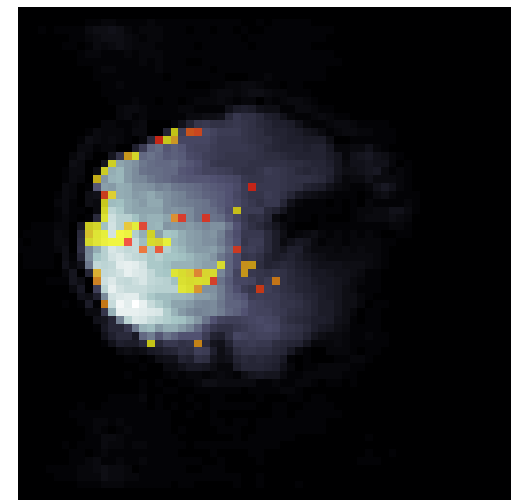

(a) Slice 3

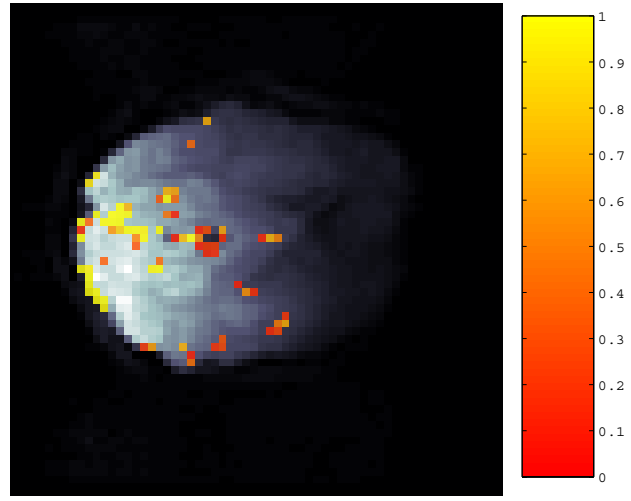

(b) Slice 5

Figure 9: Posterior probability that the increase of signal is greater than 0.01 .

Finally, in order to represent the estimated hemodynamic response function, we select some active voxels and compute the posterior mean of the estimate $\hat{y}=b+z h(\nu)$. Additionally, a $95 \%$ high posterior density interval of the estimate is computed from the samples obtained. The mean response and the 95\% interval are plotted in Figure 11, together with the observed time series. It is interesting to observe how the model is able to capture the shape of the hemodynamic response.

Figure 12 compares the ability of the TF model and the parametric model proposed in Quirós et al. (2010), to adopt the shape of the hemodynamic response. The continuous grey line corresponds to the proposed fit of the HRF, the fit given by the Poisson parameterisation of Quirós et al. (2010) is represented by the dashed grey line and the continuous black line represents the observed data time course for an active voxel (one cycle). Once again, 


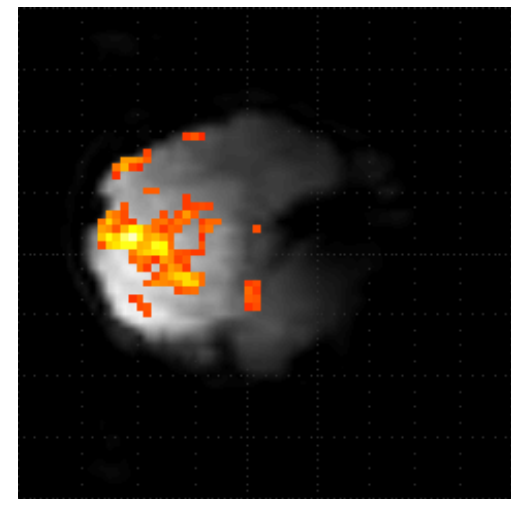

(a) Slice 3

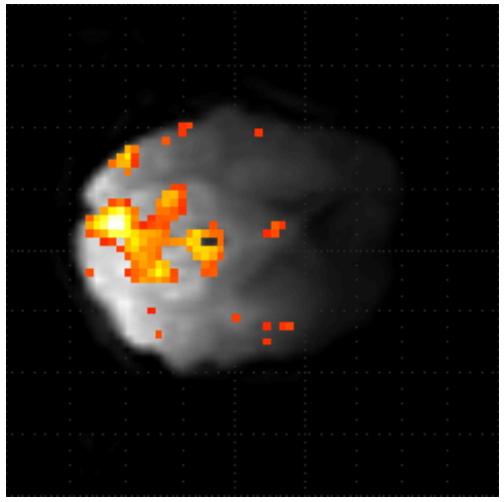

(b) Slice 5

Figure 10: Activity estimate given by SPM.

although both approaches provide accurate estimations, the model here proposed demonstrates a greater accommodation of data than Quirós et al. (2010). Notice, for instance, that the restrictions imposed by the Poisson parameterisation do not permit the curve to model a rapid increase immediately after the stimulus onset while the transfer function does and that the parametric curve is unable to define a flatter decay, as the TF shows. 

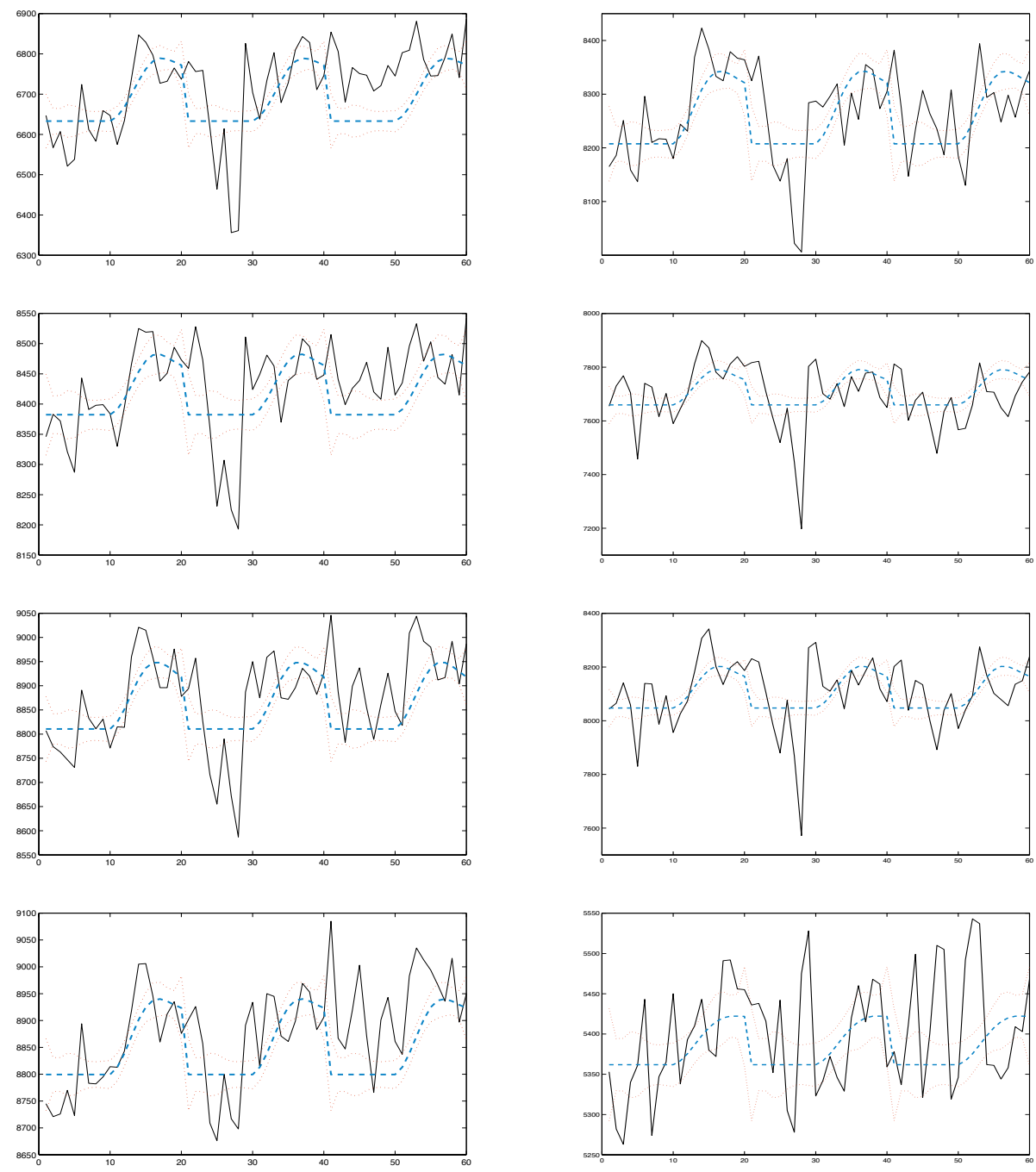

Figure 11: Mean response and the 95\% interval of the HRF fit in several active voxels. 

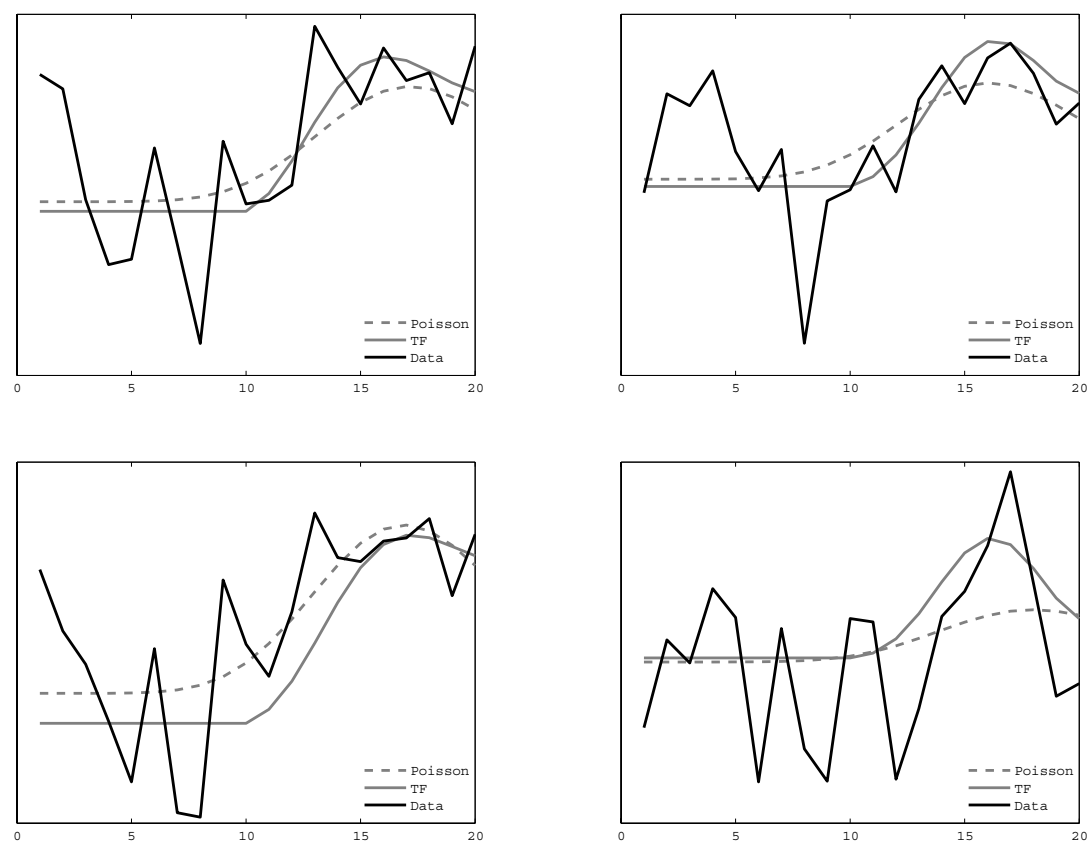

Figure 12: Estimation of the HRF for the TF and the Poisson parameterisation of Quirós et al. (2010) and one cycle of the real data. 


\subsection{Discussion}

This work describes the inclusion of transfer functions in the spatiotemporal model proposed by Quirós et al. (2010) to estimate the HRF in the analysis of fMRI data. In order to avoid overfitting and that parameters become ill determined, smoothness in the TF is imposed using a GMRF as prior distribution for the coefficients of the TF.

Using a TF for modelling the HRF has a number of advantages over standard parametric families (Poisson, gamma, Gaussian). In particular, the TF model does not impose a specific form on the hemodynamic response, leading to a greater capability of accommodating the characteristic shape of the theoretical HRF. When compared to Quirós et al. (2010), this flexibility in the temporal estimation allows us to simplify the spatial modelling of brain activation. For instance, there is no need to use a scale factor in order to accommodate the form of the Poisson density function to the hemodynamic response, resulting in an easier to implement MCMC algorithm. Overall this simplification leads to a computational improvement.

Nevertheless, in the fMRI data analysis, there is a trade-off between sensitive detection of brain activity and accurate estimation of the hemodynamic response. By using a more flexible model for the hemodynamic response, such as a transfer function approach proposed here, there is a risk of losing interpretability of the estimated parameters and sensitivity in the activity

detection. A line of future research, consequently, is to include additional spatial structure in the model. In particular, an obvious extension to this model would be to impose some spatial structure on the coefficients of the transfer function. In this way, the temporal GMRF used as prior distribution 
for $\nu$ would be extended to a spatiotemporal GMRF by adding autoregressive dependence in the spatial dimension, leading to a non-separable modelisation.

Makni et al. (2008) also propose a spatiotemporal model using TF for estimating the HRF. The benefits of our proposal with respect to this work are that: (i) In Makni et al. (2008), it is necessary to constrain the HRF to be of unitary norm to overcome the scale ambiguity problem. Such a problem does not arise in our modelisation, given that $z$ is binary. (ii) The results given by the model described in Makni et al. (2008) depend on the election of the so called parcels since the HRF shape may only vary from one parcel to another. However, we propose a modelisation in which the HRF may vary from voxel to voxel. (iii) In addition, our model includes spatial correlation through the prior for $w$, avoiding spatial smoothing during the preprocessing.

We introduce spatial correlation in the prior model for $w$ via a GMRF. Making use of GMRF as prior distribution for the parameter indicating activity presence, the activity localisation in a voxel is determined by the response magnitude in it and also by the activity evidence in its neighbouring voxels. That is, we place, a priori, the expectation that activity takes the form of regions conformed of several neighbouring voxels.

Complex noise modelisation is not taken into account in our spatiotemporal model, where $\varepsilon$ is assumed to be independent Gaussian. Future versions of our model will include more complex noise modelisation in order to deal with serial correlation, in particular we are interested in using autoregressive (AR) processes as described in Penny et al. (2003). Furthermore, it would be interesting to use an autoregressive model for the baseline parameter, $b$, 
in order to account for temporal trends and drifts in signal.

The use of Bayesian inference allows us to deal with a complex model and to include prior information about the unknown parameters of the model. An interesting area of future work is the elicitation of that information for $\kappa$ and $l$, the parameters defining the covariance matrix of the prior distribution for the coefficients of the transfer function. On the other hand, they could be included as hyperparameters of the model and estimated.

It is not an easy task to correctly infer on parameters of interest with the complexity of the model proposed, however, we are able to do it by using a fully Bayesian framework. We use MCMC algorithms to draw samples from the posterior distributions of the unknown quantities of interest. By mean of simulations, we show that this procedure is working in the right way and that the estimation is correct. The procedure is capable of reconstructing all the components of the model and estimating the corresponding hyperparameters. Furthermore, by using Bayesian inference we are able to compute the

probaility of any event of interest, as for example, the probability that the increase of signal is greater than a certain percentage.

\section{Acknowledgments}

This work was partially supported by grants from MEC (MTM200614961-C05-05, TEC2006-13966-C03-01 and TSI2007-66706-C04-01) and URJC and CAM (URJC-CM-2006-CET-0371). 


\section{References}

Bowman, F.D., 2007. Spatiotemporal Models for Region of Interest Analysis of Functional Neuroimaging Data. Journal of the American Statistical Association, 102(478): 442-453.

Box, G. and Jenkins, G., 1976. Time Series Analysis, Forecasting and Control, San Francisco: Holden-Day, second edn.

Boynton, G.M., Engel, S.A., Glover, G.H., Heeger, D.J., 1996. Linear Systems Analysis of Functional Magnetic Resonance Imaging in Human V1. The Journal of Neuroscience, 16(13): 4207-4221.

Ciuciu, P., Poline, J.-B., Marrelec, G., Idier, J., Pallier, C. and Benali, H. (2003). Unsupervised robust nonparametric estimation of the hemodynamic response function for any fmri experiment, IEEE Transactions on Medical Imaging, 22 (10): 12351251.

Friston, K.J., Jezzard, P., Turner, R., 1994. Analysis of functional MRI timeseries. Human Brain Mapping, 1(2): 153-171.

Friston, K.J., Holmes, A.P., Poline, JB., Gransby, P.J., Williams, S.C.R., Frackowiak, R.S.J., Turner, R., 1995. Analysis of fMRI time - series revisited. NeuroImage, 2(1): 45-53.

Friston, K.J., Fletcher, P., Joshephs, O., Holmes, A., Rugg, M. and Turner, R. (1998). Event-related fmri: Characterizing differential responses, NeuroImage, 7: 30-40. 
Friston, K.J., Penny, W., 2003. Posterior probability maps and SPMs. NeuroImage 19, 1240-1249.

Geweke, J., 1992. Evaluating the accuracy of sampling-based approaches to calculating posterior moments. In Bayesian Statistics 4 (ed JM Bernardo, JO Berger, AP Dawid and AFM Smith). Clarendon Press, Oxford, UK.

Gossl, C., Auer, D.P., Fahrmeir, L., 2001. Bayesian spatiotemporal inference in functional magnetic resonance imaging. Biometrics, 57(2): 554-562.

Goutte, C., Nielsen, F. A. and Hansen, L. K. (2000). Modeling the haemodynamic response in fmri using smooth fir filters, IEEE Transactions on Medical Imaging, 19 (12): 11881201.

Katanoda, K., Matsuda, Y., Sugishita, M., 2002. A Spatio-temporal Regression Model for the Analysis of Functional MRI Data. NeuroImage, 17(3): 1415-1428.

Kornak, J., 2000. Bayesian Spatial Inference from Haemodynamic Response Parameters in Functional Magnetic Resonance Imaging. PhD. Thesis, University of Nottingham.

Lange, N., Zeger, S.L., 1997. Non - Linear Fourier Time Series Analysis for Human Brain Mapping by Functional Magnetic Resonance Imaging. Applied Statistics, 46(1): 1-29.

Makni, S., Idier, J., Vincent, T., Thirion, B., Dehaene-Lambertz, G., Ciuciu, P., 2008. A fully Bayesian approach to the parcel-based detectionestimation of brain activity in fMRI. NeuroImage, 41: 941-969. 
Nielsen, F. A., Hansen, L. K., Toft, P., Goutte, C., Lange, N., Strother, S. C., Mrch, N., Svarer, C., Savoy, R., Rosen, B., Rostrup, E. and Born, P. (1997). Comparison of two convolution models for fmri time series, in NeuroImage, edited by Friberg, L., Gjedde, A., Holm, S., Lassen, N. A. and Novak, M., vol. 5, p. S473, San Diego: Academic Press, From the THIRD INTERNATIONAL CONFERENCE ON FUNCTIONAL MAPPING OF THE HUMAN BRAIN.

Ogawa, S., Lee, T.M., Kay, A.R., Tank, D.W., 1990. Functional brain mapping by blood oxygenation level - dependent contrast magnetic resonance imaging. A comparison of signal characteristics with a biophysical model. Biophysical Journal, 64: 803-12.

Penny, W., Kiebel, S., Friston, K., 2003. Variational Bayesian inference for fMRI time series. NeuroImage 19, 727-741.

Penny, W.D., Trujilllo-Barreto, N.J., Friston, K.J., 2005. Bayesian fMRI time series analysis with spatial priors. NeuroImage, 24(2): 350-362.

Quirós, A., Montes, R., Hernández, J.A., 2006. A fully Bayesian two - stage model for detecting brain activity in fMRI. Lecture Notes in Computer Science, 4345: 334-345.

Quirós, A., Montes Diez, R. and Gamerman, D. (2010). Bayesian spatiotemporal model of fMRI data, Neuroimage, 49: 442-456.

Rue, H., Held, L., 2005. Gaussian Markov Random Fields: Theory and Applications. Monographs on Statistics and Applied Probability, 104, Chapman \& Hall/CRC, London. 
Woolrich, M.W., Jenkinson, M., Brady, J.M., Smith, S.M., 2004a. Fully bayesian spatio - temporal modeling of fMRI data. IEEE Transactions on Medical Imaging, 23(2): 213-231.

Woolrich, M.W., Behrens, T.E.J., Smith, S.M., 2004b. Constrained linear basis sets for HRF modelling using variational Bayes. NeuroImage, 21(4): 1748-1761. 